\title{
ACCURACY AND RELIABILITY CONSIDERATIONS OF Option Pricing Algorithms
}

\section{YUE-KUEN KWOK* \\ KA-WO LAU}

A wide variety of computational schemes have been proposed for the numerical valuation of various classes of options. Experiences in numerical computation have revealed that the details of the implementation of the auxiliary conditions in the numerical algorithms may have profound effects on numerical accuracy. Difficulties in designing algorithms that deal with the path-dependent payoffs, monitoring features, etc., have been well reported in the literature. In this article, the theoretical issues on the assessment of numerical schemes with regard to accuracy of approximation of auxiliary conditions, rate of convergence, and oscillation phenomena are reviewed. In particular, the oscillation phenomena in bond-price calculations and the intricacies in implementing the auxiliary conditions in barrier options, proportional step options, and lookback options are discussed. With different types of options and modes of monitoring (continuous or discrete), the optimal method of placing the lattice nodes with reference to

\footnotetext{
${ }^{*}$ Correspondence author, Department of Mathematics, Hong Kong University of Science and Technology, Clear Water Bay, Hong Kong; e-mail: maykwok@ust.hk

Received February 1997; Accepted January 2001
}

- Yue-Kuen Kwok is in the Department of Mathematics at Hong Kong University of Science and Technology in Clear Water Bay, Hong Kong.

- Ka-Wo Lau is in the Department of Computer Sciences at Hong Kong University of Science and Technology in Clear Water Bay, Hong Kong. 
the boundary (absorbing or reflecting) are examined in order to achieve linear temporal rate of convergence. (C) 2001 John Wiley \& Sons, Inc. Jrl Fut Mark 21:875-903, 2001

\section{INTRODUCTION}

The option-pricing model initiated by Black and Scholes (1973) led to a parabolic partial differential equation. The individual contractual specifications are distinguished by the auxiliary conditions in the option models. From the arbitrage-option-pricing theory, the option value can be interpreted as the discounted expectation value of the terminal payoff in the risk-neutralized world. However, only a few of the option models lend themselves to closed-form pricing formulas. Even with the availability of these so-called analytic formulas, they often are expressed in terms of multivariate cumulative normal distribution functions. The valuation of the analytic formulas normally requires some numerical procedures. For options with more-exotic payoffs, one must resort to numerical algorithms for their valuation. Nowadays, it becomes quite common to demand the computation of thousands of option values within a short duration of time. This prompts the necessity to develop numerical algorithms for option valuation that are competitive in terms of accuracy, efficiency, and reliability.

Roughly speaking, option valuation algorithms can be classified into three categories. The analytic-approximation approach seeks a simpler approximating model whose value gives a well-acceptable approximation to that of the original option model. The main advantages are that comparatively simpler valuation procedures are required to obtain the approximation values. In American-option models, the prominent examples are the compound option-approximation method (Geske \& Johnson, 1984), the quadratic-approximation method (MacMillan, 1986), and the recursive-integration method (Huang, Subrahmanyam, \& Yu, 1996). Other examples include the approximation formulas for the discretely monitored barrier options and lookback options (Broadie, Glasserman, \& Kou, 1999). The derivation of these approximation formulas invariably require sophisticated analytic techniques. At the other end of the spectrum, one may obtain an estimate of the price of a newly structured option through Monte Carlo simulation. The Monte Carlo method is basically a numerical procedure for estimating the expected value of a random variable, so it leads itself naturally to option-pricing problems represented as expectations. Monte Carlo simulation is invariably 
computationally demanding, but its efficiency can be enhanced greatly through the use of various variance reduction techniques (Boyle, Broadie, \& Glasserman, 1997).

Between the analytic-approximation methods and the Monte Carlo simulation lie the tree methods (binomial/trinomial schemes) and the finite difference methods. Collectively, they are called the lattice methods because option values at the lattice nodes are calculated in the numerical procedures. The tree methods simulate the asset price process by a discrete random-walk model, and the discrete process converges to the continuous lognormal diffusion as the time step tends to zero (Cox, Ross, \& Rubinstein, 1979). The finite difference approach seeks the discretization of the differential operators in the governing equation of the continuousoption model. Indeed, the tree methods and the finite difference methods are related closely, though they are derived based on different approaches. The tree methods can be considered as members in the class of explicit finite difference schemes. The amount of computation time required and the algorithmic development efforts demanded for the tree methods and the finite difference methods somewhat lie between the analyticapproximation approach and the Monte Carlo simulation.

The literature on the construction and analysis of option-pricing algorithms using the lattice approach is quite voluminous. The articles by AitSahlia and Carr (1997) and Broadie and Detemple (1997) list detailed comparisons of the performance of various option-pricing algorithms. It is known widely that accuracy of numerical-option values may deteriorate due to the proximity of the asset price to the barrier (Boyle \& Tian, 1998; Steiner \& Wallmeier, 1999) and the discontinuity of the derivative of the terminal payoff at the strike price (Heston \& Zhou, 2000; Tian, 1999).

This article addresses some of the theoretical issues on the assessment of the tree methods and the finite difference schemes. These schemes are analyzed with reference to accuracy of approximation of auxiliary conditions, rate of convergence, oscillation phenomena of computation, etc. The article is organized as follows. In the next section, the different approaches used for the construction of numerical algorithms in the literature are summarized, and their similarities and differences are addressed. The coefficients in all numerical schemes are shown to be dependent on two parameters. The modified equivalent partial differential equation is used to analyze the order of accuracy of numerical schemes. These analyses shed light on the issue on the choice of optimal parameter in trinomial schemes. In the following section, the linkage of 
the non-negativity of probability values in the tree methods with the oscillation phenomena associated with numerical computation is examined. The sample calculations on pricing a bond are used to illustrate how to avoid oscillation phenomena in numerical calculations. Then the results are presented of numerical experiments with the barrier options, proportional step option, and lookback option to reveal the impact of approximation of auxiliary conditions on the overall rate of convergence of numerical calculations. Some theoretical explanations to the success of certain methods of approximation of auxiliary conditions are offered. The article ends with summaries of results and conclusions.

\section{RATE OF CONVERGENCE OF NUMERICAL SCHEMES}

The Black-Scholes pricing model is a continuous model where the continuous lognormal diffusion process is assumed for the asset price movement. By following the replicating-portfolio approach, Cox et al. (1979) developed the renowned discrete binomial pricing model, in which the asset price is allowed to have binomial jumps over one time period. Their binomial model still enjoys great popularity in the finance community for numerical valuation of a variety of option models, due primarily to its ease of implementation and pedagogical appeal. The jump probabilities in the binomial model are determined by matching the mean and variance of the asset price ratio over one period in both continuous and discrete models.

\section{Trinomial Schemes}

By allowing a three-jump process for the asset price movement, it was envisioned that the availability of an additional parameter in a trinomial model leads to a numerical scheme that may converge faster to the continuous solution. The additional flexibility is reflected in the ratio of the time step to the square of the logarithm of the jump ratio, which is not necessarily fixed in the trinomial models. Boyle (1988) obtained the parameter values in the trinomial model by matching the mean and variance of the asset price ratio over one period. Tian (1993) determined the trinomial parameters by matching higher-order moments of the asset price ratio. Since the mean and variance of the asset price ratio involve exponential terms, the algebraic expressions of the parameters in their trinomial models are quite cumbersome. The situation becomes worse when Boyle (1988) extended the trinomial jump process to multi-asset option models. Since the logarithm of the asset price ratio follows a 
normal distribution, a more sensible approach should match the mean and variance of the logarithm of the asset price ratio process.

Let $\Delta t$ be the time step and write $\Delta x=\ln u=-\ln d$, where $u$ and $d$ are the upward-and downward-jump ratios of the asset price $S$. Let $V_{j}^{n}$ denote the option value at the trinomial node, $n$ time steps from expiry, and $j$ upward jumps from the current asset price. The trinomial scheme takes the form:

$$
V_{j}^{n+1}=\left(b_{1} V_{j+1}^{n}+b_{0} V_{j}^{n}+b_{-1} V_{j-1}^{n}\right) e^{-r \Delta t},
$$

where $r$ is the riskless interest rate. Since $x=\ln S$ follows the Brownian process, the appropriate relative order of magnitude of differential change of time $\Delta t$ and differential diffusion distance $\Delta x$ in a Brownian process should observe

$$
\Delta x^{2} \sim \Delta t
$$

It may be written as

$$
\Delta x^{2}=\lambda^{2} \sigma^{2} \Delta t
$$

where $\sigma$ is the volatility of the asset price process and $\lambda$ is a free parameter. Next defined is

$$
\mu=\sigma^{2} \frac{\Delta t}{\Delta x^{2}} \quad \text { and } \quad c=\left(r-\frac{\sigma^{2}}{2}\right) \frac{\Delta t}{\Delta x}
$$

where $\mu$ and $c$ are called the volatility parameter and the drift parameter ( $c$ is commonly called the Courant number in numerical analysis literature), respectively. It will be illustrated later that all option-pricing schemes for one-asset option models can be expressed solely in terms of these two parameters. Furthermore, one may observe from eqs. (2b) and (3) that $\mu=\frac{1}{\lambda^{2}}$; so if $\lambda^{2}=O(1)$, then $c=O(\sqrt{\Delta t})$.

\section{Lax-Wendroff Scheme}

By equating the mean and variance of the logarithm of the asset price ratio over one time period, the following linear system of equations for $b_{1}$ and $b_{-1}$ is obtained:

$$
\begin{aligned}
& \left(b_{1}-b_{-1}\right) \Delta x=\left(r-\frac{\sigma^{2}}{2}\right) \Delta t \\
& \left(b_{1}+b_{-1}\right) \Delta x^{2}=\sigma^{2} \Delta t+\left(r-\frac{\sigma^{2}}{2}\right)^{2} \Delta t^{2} .
\end{aligned}
$$


The remaining probability value, $b_{0}$, is obtained from the relation:

$$
b_{0}=1-\left(b_{1}+b_{-1}\right) .
$$

Solving the above equations for $b_{-1}, b_{0}$, and $b_{1}$, the following LaxWendroff scheme (Lax \& Wendroff, 1960) is obtained:

$$
V_{j}^{n+1}=\left[\frac{c^{2}+\mu+c}{2} V_{j+1}^{n}+\left(1-c^{2}-\mu\right) V_{j}^{n}+\frac{c^{2}+\mu-c}{2} V_{j-1}^{n}\right] e^{-r \Delta t} .
$$

\section{Kamrad-Ritchken Scheme}

Kamrad and Ritchken (1991) dropped the $\Delta t^{2}$ term in eq. (4b) as an approximation, so their trinomial scheme can never achieve $O\left(\Delta t^{2}\right)$ accuracy with any choice of $\lambda$. The Kamrad-Ritchken scheme can be expressed as

$$
V_{j}^{n+1}=\left[\frac{\mu+c}{2} V_{j+1}^{n}+(1-\mu) V_{j}^{n}+\frac{\mu-c}{2} V_{j-1}^{n}\right] e^{-r \Delta t} .
$$

\section{Finite Difference Methods}

In the Black-Scholes model, the lognormal diffusion process is assumed for the asset price movement. This dictates $x=\ln S$ to be the better choice of the independent variable in the model, which then leads naturally to a constant coefficient equation governing the option value. More importantly, the logarithmic transformation leads to better numerical stability in the calculations [the transformation results in flattening of the eigenvalues in the discretization matrix, see Tavella (2000) for more details]. Also, the use of $S$ as the independent variable may lead to numerical oscillations [see Spurious Oscillations of Computed Solutions and eq. (26)]. We let $v(x, \tau)=e^{r \tau} V(S, \tau)$, where $V(S, \tau)$ denotes the option price and $\tau$ is the time to expiry. The Black-Scholes equation for an one-asset option model becomes

$$
\frac{\partial v}{\partial \tau}=\frac{\sigma^{2}}{2} \frac{\partial^{2} v}{\partial x^{2}}+\left(r-\frac{\sigma^{2}}{2}\right) \frac{\partial v}{\partial x}
$$

Let the domain of interest be $(L, U) \times(0, T]$, and write $v_{j}^{n}$ as the numerical approximation of $v(x, t)$ at the $(j, n)$ th node in the computational mesh. In the finite difference mesh, the $(j, n)$ th node refers to the point $(L+j \Delta x, n \Delta t)$ in the $x-\tau$ plane, where $\Delta x$ and $\Delta t$ are the stepwidth and the time step, respectively. 
The most straightforward approach to derive finite difference schemes is the use of finite difference operators to approximate the differential operators in eq. (7). Suppose the following differential operators are used

$$
\begin{aligned}
\frac{\partial v}{\partial \tau}(L+j \Delta x, n \Delta t) & =\frac{v_{j}^{n+1}-v_{j}^{n}}{\Delta t}+O(\Delta t) \\
\frac{\partial v}{\partial x}(L+j \Delta x, n \Delta t) & =\frac{v_{j+1}^{n}-v_{j-1}^{n}}{2 \Delta x}+O\left(\Delta x^{2}\right) \\
\frac{\partial^{2} v}{\partial x^{2}}(L+j \Delta x, n \Delta t) & =\frac{v_{j+1}^{n}-2 v_{j}^{n}+v_{j-1}^{n}}{\Delta x^{2}}+O\left(\Delta x^{2}\right),
\end{aligned}
$$

the corresponding finite difference scheme is called the Forward-Time Centered-Difference Scheme. Interestingly, this numerical scheme takes exactly the same form as the Kamrad-Ritchken trinomial scheme.

\section{Crank-Nicolson Scheme}

Crank and Nicolson (1947) approximated the spatial-differential operators in eq. (7) by the average of the centered-difference operators in eqs. $(8 \mathrm{~b}, \mathrm{c})$ evaluated at the middle of the $n$th and $(n+1)$ th time levels. This results the following two-level six-point implicit scheme

$$
\begin{gathered}
\frac{-\mu-c}{4} v_{j+1}^{n+1}+\left(1+\frac{\mu}{2}\right) v_{j}^{n+1}+\frac{-\mu+c}{4} v_{j-1}^{n+1} \\
=\frac{\mu+c}{4} v_{j+1}^{n}+\left(1-\frac{\mu}{2}\right) v_{j}^{n}+\frac{\mu-c}{4} v_{j-1}^{n} .
\end{gathered}
$$

\section{Order of Accuracy}

All the above trinomial schemes and finite difference schemes can be recast into the following two-level six-point form:

$$
a_{-1} v_{j-1}^{n+1}+a_{0} v_{j}^{n+1}+a_{1} v_{j+1}^{n+1}=b_{-1} v_{j-1}^{n}+b_{0} v_{j}^{n}+b_{1} v_{j+1}^{n},
$$

where $a_{-1}, a_{0}, a_{1}, b_{-1}, b_{0}$, and $b_{1}$ are coefficients in the numerical scheme. Since only derivative terms appear in eq. (7), a trivial solution for $v(x, \tau)$ is the constant solution, so the minimal condition for consistency is given by

$$
a_{-1}+a_{0}+a_{1}=b_{-1}+b_{0}+b_{1}=1 .
$$


Note that there are only four independent parameters in the numerical scheme (10). For convenience, it is defined

$$
\begin{aligned}
& a_{s}=a_{1}+a_{-1}, \quad a_{d}=a_{1}-a_{-1}, \\
& b_{s}=b_{1}+b_{-1} \quad \text { and } \quad b_{d}=b_{1}-b_{-1} .
\end{aligned}
$$

When both $a_{-1}$ and $a_{1}$ are non-zero, the solution of the numerical scheme eq. (12) requires the inversion of a tridiagonal system of equations in every time step. In this case, the scheme is termed implicit since $v_{j}^{n+1}$ cannot be obtained explicitly. On the other hand, the scheme becomes explicit when both $a_{-1}=a_{1}=0$. With the use of the efficient Thomas algorithm (Thomas, 1995), the operational counts for solving an implicit two-level six-point scheme are less than double those for an explicit two-level four-point scheme.

\section{Modified Equivalent Partial Differential Equation}

Since the numerical scheme is a discretization of the governing differential equation, the solution to the two-level six-point scheme (10) does not satisfy exactly the differential equation, but rather only to some order of accuracy (precise definition of order of accuracy to be given later). The modified equivalent partial differential equation (MEPDE) of a given numerical scheme is defined to be the differential equation whose solution is the same as that of the numerical scheme.

The MEPDE provides an effective tool to assess the order of accuracy of scheme (10) for solving eq. (7). The MEPDE is derived by performing the Taylor-series expansion of each term in the numerical scheme (10) about the $(j, n)$ th grid point, and then eliminating the time derivatives higher than the first order, and all mixed time and space derivatives. The MEPDE of the numerical scheme (10) is known to be (Kwok, 1992).

$$
\begin{aligned}
\frac{\partial v}{\partial \tau}= & \left(b_{d}-a_{d}\right) \frac{\Delta x}{\Delta t} \frac{\partial v}{\partial x}+\frac{\left(b_{s}-a_{s}\right)-\left(b_{d}^{2}-a_{d}^{2}\right)}{2} \frac{\Delta x^{2}}{\Delta t} \frac{\partial^{2} v}{\partial x^{2}} \\
& +\frac{\left(b_{d}-a_{d}\right)+3\left(a_{d} a_{s}-b_{d} b_{s}\right)+2\left(b_{d}^{3}-a_{d}^{3}\right)}{6} \frac{\Delta x^{3}}{\Delta t} \frac{\partial^{3} v}{\partial x^{3}} \\
& +\frac{2\left(a_{d}^{2}-b_{d}^{2}\right)-\left(a_{s}-b_{s}\right)\left[1-3\left(a_{s}+b_{s}\right)+4\left(a_{d}^{2}+a_{d} b_{d}+b_{d}^{2}\right)\right]}{24} \frac{\Delta x^{4}}{\Delta t} \frac{\partial^{4} v}{\partial x^{4}} \\
& + \text { higher order spatial derivative terms }
\end{aligned}
$$


A numerical scheme is said to be $m$ th order space accurate if its corresponding MEPDE agrees with the given partial differential equation up to the $m$ th order spatial derivatives.

The order of accuracy of some of the above schemes then is assessed by substituting the appropriate parameters into eq. (13). The corresponding MEPDE's are found to be:

Lax-Wendroff Scheme

$$
\begin{aligned}
\frac{\partial v}{\partial \tau}= & \left(r-\frac{\sigma^{2}}{2}\right) \frac{\partial v}{\partial x}+\frac{\sigma^{2}}{2} \frac{\partial^{2} v}{\partial x^{2}}+\frac{(1-3 \mu) c-c^{3}}{6} \frac{\Delta x^{3}}{\Delta t} \frac{\partial^{3} v}{\partial x^{3}} \\
& +\frac{\mu-3 \mu^{2}-2 \mu c^{2}+c^{4}-c^{2}}{24} \frac{\Delta x^{4}}{\Delta t} \frac{\partial^{4} v}{\partial x^{4}}+\cdots
\end{aligned}
$$

Kamrad-Ritchken Scheme

$$
\begin{aligned}
\frac{\partial v}{\partial \tau}= & \left(r-\frac{\sigma^{2}}{2}\right) \frac{\partial v}{\partial x}+\left[\frac{\sigma^{2}}{2}-\frac{\left(r-\frac{\sigma^{2}}{2}\right)^{2}}{2} \Delta t\right] \frac{\partial^{2} v}{\partial x^{2}} \\
& +\frac{(1-3 \mu) c+2 c^{3}}{6} \frac{\Delta x^{3}}{\Delta t} \frac{\partial^{3} v}{\partial x^{3}} \\
& +\frac{\mu+4 \mu c^{2}-3 \mu^{2}-2 c^{2} \Delta x^{4}}{\Delta t} \frac{\partial^{4} v}{\partial x^{4}}+\cdots
\end{aligned}
$$

Crank-Nicolson Scheme

$$
\begin{aligned}
\frac{\partial v}{\partial \tau}= & \left(r-\frac{\sigma^{2}}{2}\right) \frac{\partial v}{\partial x}+\frac{\sigma^{2}}{2} \frac{\partial^{2} v}{\partial x^{2}}+\frac{\left(2+c^{2}\right) c}{12} \frac{\Delta x^{3}}{\Delta t} \frac{\partial^{3} v}{\partial x^{3}} \\
& +\left(1+c^{2}\right) \mu \frac{\Delta x^{4}}{\Delta t} \frac{\partial^{4} v}{\partial x^{4}}+\cdots
\end{aligned}
$$

Several observations and conclusions on the order of accuracy of the numerical schemes can be drawn from the analyses of the above MEPDE's. All MEPDE's tend to the Black-Scholes equation as $\Delta t \rightarrow 0$ and $\Delta x \rightarrow 0$. Unlike the other schemes, the coefficient of $\frac{\partial^{2} v}{\partial x^{2}}$ in the MEPDE of the Kamrad-Ritchken scheme is not equal to $\frac{\sigma^{2}}{2}$ exactly. However, the coefficient does tend to $\frac{\sigma^{2}}{2}$ as $\Delta t \rightarrow 0$. This is attributed to the deliberate dropping of $O\left(\Delta t^{2}\right)$ term in the derivation procedure of the Kamrad-Ritchken scheme [see eq. (4b)]. 
Recall that one should choose $\Delta t \sim \Delta x^{2}$ in order to represent the Brownian process of $\ln S$ appropriately [see eqs. (2a,b)]. If $\mu=O(1)$ and $c=O(\sqrt{\Delta t})$ is treated, then the coefficients of derivative terms beyond the second order in the above MEPDE's are $O(\Delta t)$. Note that for the Lax-Wendroff scheme, when $\mu$ is chosen to be $1 / 3$ (equivalently, $\lambda=\sqrt{3})$, the coefficients of the third and higher order derivatives become $O\left(\Delta t^{2}\right)$.

One may be tempted to popularize the use of second-order timeaccurate schemes in option-pricing calculations. However, the overall order of accuracy of a numerical algorithm also is affected by the continuity properties of the initial conditions, the order of accuracy in the approximation of the boundary conditions. In most option-pricing calculations, second-order temporal accuracy is often degraded to first order because of the discontinuity in the first-order derivative of the terminal payoff functions, first-order boundary value approximation, etc. (see Barrier-Type Option Models and Figs. 2-5).

\section{SPURIOUS OSCILLATIONS OF COMPUTED SOLUTIONS}

A more succinct argument on the relations of the non-negativity of the coefficients in the numerical schemes and the spurious oscillations of the computed solutions are revealed as follows. The discussion of oscillation phenomena in the numerical calculations of barrier and Asian pricing models can be found in Zvan, Vetzal, and Forsyth's articles (1998a, 1998b, 2000).

\section{Theorem}

Suppose the coefficients in the following explicit scheme

$$
v_{j}^{n+1}=b_{-1} v_{j-1}^{n}+b_{0} v_{j}^{n}+b_{1} v_{j+1}^{n}
$$

are non-negative, and the initial values are bounded, that is $\max _{j}\left|v_{j}^{0}\right| \leq M$ for some constant $M$; then

$$
\max _{j}\left|v_{j}^{n}\right| \leq M, \text { for all } n \text {. }
$$

\section{Proof}

From eq. (7), it is deduced that

$$
\left|v_{j}^{n+1}\right| \leq\left|b_{-1}\right|\left|v_{j-1}^{n}\right|+\left|b_{0}\right|\left|v_{j}^{n}\right|+\left|b_{1}\right|\left|v_{j+1}^{n}\right|,
$$


so

$$
\begin{aligned}
\max _{j}\left|v_{j}^{n+1}\right| & \leq\left|b_{-1}\right| \max _{j}\left|v_{j-1}^{n}\right|+\left|b_{0}\right| \max _{j}\left|v_{j}^{n}\right|+\left|b_{1}\right| \max _{j}\left|v_{j+1}^{n}\right| \\
& =b_{-1} \max _{j}\left|v_{j-1}^{n}\right|+b_{0} \max _{j}\left|v_{j}^{n}\right|+b_{1} \max _{j}\left|v_{j+1}^{n}\right|
\end{aligned}
$$

because $b_{-1}, b_{0}$ and $b_{1}$ are non-negative. Let $E^{n}=\max _{j}\left|v_{j}^{n}\right|$, the above inequality can be transformed into

$$
E^{n+1} \leq b_{-1} E^{n}+b_{0} E^{n}+b_{1} E^{n}=E^{n} \text { since } \quad b_{-1}+b_{0}+b_{1}=1 .
$$

Deductively,

$$
E^{n} \leq E^{n-1} \leq \cdots \leq E^{0}=\max _{j}\left|v_{j}^{0}\right| \leq M .
$$

can be obtained.

What would happen when one or more of the coefficients of the explicit scheme eq. (7) are negative? If $b_{0}<0, b_{-1}>0$ and $b_{1}>0$, and let $v_{0}^{0}=\varepsilon>0$ and $v_{j}^{0}=0, j \neq 0$. At the next time level, $v_{-1}^{1}=b_{1} \varepsilon$, $v_{0}^{1}=b_{0} \varepsilon, v_{1}^{1}=b_{-1} \varepsilon$, where the sign of $v_{j}^{1}$ alternates with $j$. The alternating sign property can be shown to persist at all later time steps. Hence, it can deduced from eq. (7) that

$$
\left|v_{j}^{n+1}\right|=b_{-1}\left|v_{j-1}^{n}\right|-b_{0}\left|v_{j}^{n}\right|+b_{1}\left|v_{j+1}^{n}\right| .
$$

Define $S^{n}=\Sigma_{j}\left|v_{j}^{n}\right|$ and sum over all values of $j$ of the above identity. Now,

$$
\sum_{j}\left|v_{j}^{n+1}\right|=b_{-1} \sum_{j}\left|v_{j-1}^{n}\right|-b_{0} \sum_{j}\left|v_{j}^{n}\right|+b_{1} \sum_{j}\left|v_{j+1}^{n}\right|
$$

or

$$
S^{n+1}=\left(b_{-1}-b_{0}+b_{1}\right) S^{n}=\left(1-2 b_{0}\right) S^{n} .
$$

Note that $1-2 b_{0}>1$ since $b_{0}<1$. Deductively,

$$
S^{n}=\left(1-2 b_{0}\right)^{n} S^{0}=\left(1-2 b_{0}\right)^{n} \varepsilon
$$

is obtained, and as $n \rightarrow \infty, S^{n} \rightarrow \infty$. The above result reveals the oscillation phenomena where the solution values oscillate in signs at neighboring nodes, and the magnitudes grow with increasing number of time steps.

\section{Bond Price Calculations}

In most equity option calculations, if $x=\ln S$ is used as the state variable, the coefficients in the finite difference schemes or trinomial schemes normally observe the non-negativity condition. This is because 
$\mu=O(1)$ (with $\mu<1$ ) and $c=O(\sqrt{\Delta t}$ ) is chosen so that $\mu>|c|$ is satisfied. However, if the volatility $\sigma$ is a function of the state variable having the property that $\sigma$ tends to zero as the value of the state variable goes to zero, then negative coefficient in the numerical schemes may be encountered.

As an example, consider the numerical calculation of the price of a bond $B(r, \tau)$, where the interest rate $r$ is governed by the CIR interest rate process

$$
d r=\alpha(\beta-r) d t+\gamma \sqrt{r} d \mathrm{Z}
$$

where $\alpha, \beta$, and $\gamma$ are constant parameter values and $d \mathrm{Z}$ is the Wiener process. The volatility of the process is $\gamma \sqrt{r}$, which tends to zero as $r \rightarrow 0$. Oscillation phenomena occur when calculations are performed within the range of $r$ such that the coefficients in the numerical schemes become negative. One may avoid the occurrence of negative coefficients by restricting a lower bound $r_{\min }$ on the range of $r$.

In Table I, the numerical bond values at various interest rate values obtained with different choices of lower bound $r_{\text {min }}$ of the computational domain $\left[r_{\text {min }}, r_{\text {max }}\right] \times[0, T]$ are listed, where $r_{\max }=1.2$ and $\tau=1.0$. The interest rate follows the CIR process: $d r=0.48(0.11-r) d t+0.01 \sqrt{r} d Z$. The bond values obtained from the analytical bond-price formula also are included for reference. When $r_{\text {min }} \leq 0.02$, oscillation phenomena at small values of $r$ is observed.

The terminal payoff function of other interest-rate instruments, like a bond option, commonly possesses discontinuity in the first-order derivative. This is unlike a bond whose terminal payoff is a constant function. It is expected that the above oscillation phenomena would become more severe when the same numerical scheme is applied to the pricing of

TABLE I

Comparison of Numerical Bond Values Obtained from Finite-Difference Calculations with Different Lower Bounds, $r_{\min }$

\begin{tabular}{lccccc}
\hline & $r=0.04$ & $r=0.045$ & $r=0.05$ & $r=0.055$ & $r=0.06$ \\
\hline$r_{\text {min }}=0.03$ & 0.384 & 0.380 & 0.376 & 0.373 & 0.369 \\
$r_{\text {min }}=0.025$ & 0.385 & 0.380 & 0.377 & 0.373 & 0.369 \\
$r_{\text {min }}=0.02$ & 0.376 & 0.383 & 0.376 & 0.373 & 0.369 \\
$r_{\text {min }}=0.015$ & 0.131 & 0.440 & 0.366 & 0.374 & 0.369 \\
Analytical solution & 0.385 & 0.381 & 0.377 & 0.373 & 0.369 \\
\hline
\end{tabular}

The solution values oscillate at small values of $r$ when $r_{\min } \leq 0.02$. 
more exotic types of interest-rate instruments. Also, the oscillatory nature of numerical solutions would become even worse when the hedging parameters of derivative values are computed.

Suppose $S$ instead of $x=\ln S$ is used as the state variable in the finite difference scheme, the corresponding diffusion parameter $\mu$ and drift parameter $c$ then become

$$
\mu=\sigma^{2} S^{2} \frac{\Delta t}{\Delta S^{2}} \quad \text { and } \quad c=r S \frac{\Delta t}{\Delta S} .
$$

Consequently, oscillation phenomena is encountered when $\mu<c$, which occur as $S$ assumes small values.

The boundary condition of the bond-price model at zero interest rate cannot be deduced readily. Büttler (1995) observed that the numerical accuracy of the bond price computed depends sensibly on the choice of the boundary-approximation scheme applied at $r=r_{\min }$. The prescription of an inaccurate numerical-boundary condition would deteriorate the overall accuracy of the numerical solutions at interior node points. Rather than imposing an artificial numerical boundary condition, one may discretize the governing equation at the boundary nodes using onesided difference operators to approximate the differential operators [see Vetzal, 1998, and eqs. (28a,b)].

\section{APPROXIMATION OF THE AUXILIARY CONDITIONS}

The individual contractual specifications of different types of pathdependent options are distinguished by the auxiliary conditions imposed on the pricing models. It is well known in the literature that the naïve implementation of the auxiliary conditions into the algorithms invariably lead to erratic behaviors of the computed solutions. Even the numerical results converge to the desired solutions, the convergence rate may be painfully slow.

\section{Erratic Pattern of Convergence}

It is well reported in the literature that the numerical option values obtained from the trinomial or finite difference calculations commonly exhibit wavy or erratic pattern of convergence to the analytical solutions. Heston and Zhou (2000) demonstrated that the rate of convergence of binomial/trinomial calculations fluctuate between $O(\sqrt{\Delta t})$ and $O(\Delta t)$ [equivalent to $O\left(\frac{1}{\sqrt{N}}\right)$ and $O\left(\frac{1}{N}\right)$, where $N$ is the total number of time 
steps]. The common extrapolation technique for the enhancement of the rate of convergence cannot be applied to numerical option values due to the lack of smooth convergence.

The non-smoothness of convergence is attributed to the nondifferentiability of the terminal payoff function and improper treatment of numerical boundary conditions with reference to the path-dependent feature. Figlewski and Gao (1999) presented the adaptive-mesh trinomial model in which most part of the tree is set up with relatively coarse steps, but sections of higher-resolution lattice are grafted on in areas close to maturity and barriers. Tian (1999) proposed a simple technique of positioning nodes exactly on the strike price and the barrier. Heston and Zhou (2000) proposed two simple smoothness-enhancement techniques, namely, an adjustment of the discrete-time solution prior to maturity and smoothness of the payoff function.

In the following sample calculations on a continuous-barrier option, it is demonstrated that the proper treatment of the boundary condition (at the barrier and the far field) may increase the rate of convergence of the numerical scheme beyond the first order.

\section{Barrier-Type Option Models}

There are two major factors that lead to the deterioration of accuracy of barrier-option calculations; one is the positioning of the nodes relative to the barrier, and the other is the proximity of the initial asset price to the barrier.

\section{Positioning of the Nodes Relative to the Barrier}

Several studies have reported that the best numerical solutions are obtained if the barrier is placed to pass through a layer of nodes for the continuously monitored barrier options, and is located halfway between two layers of nodes for the discretely monitored barrier options (Boyle \& Tian, 1998; Cheuk \& Vorst, 1996; Tian, 1997). The reasons why these two different methods of placing the barriers are needed are due to the different formulations of the barrier conditions regarding the nature of monitoring.

When the barrier is monitored discretely, the barrier option resembles a vanilla option at times not at a monitoring instant. At the monitoring instants, the option value becomes zero when the asset price falls within the knock-out region. On the other hand, when the barrier is monitored continuously, the effect of the barrier is enforced at all times. 
By applying analytic continuation of the option-price function into the knock-out region, the option-price function of a continuously monitored barrier option almost observes the odd extension property when $S$ stays close to $B$, that is, $V(S, \tau) \approx-V\left(\frac{B^{2}}{S}, \tau\right)$, where $B$ is the constant barrier level and $V$ is the barrier option value.

Suppose the approach of finite volume discretization is employed to interpret the finite difference scheme; one visualizes the nodal value $v_{j}^{n}$ as representing the average of the option value within the interval $\left[x_{j}-\frac{\Delta x}{2}, x_{j}+\frac{\Delta x}{2}\right]$ at $\tau=n \Delta t$. Let $x_{b}=\ln B$, which is the position of the down-and-out barrier in terms of the transformed variable $x=\ln S$. An explanation is provided for the different methods of positioning the barrier under the two forms of monitoring of the barrier.

First, the discretely monitored barrier options are considered. Suppose the node $x_{0}$ is placed exactly on $x_{b}$, then $v_{0}^{n}$ should approximate the average of the option value within $\left[x_{b}-\frac{\Delta x}{2}, x_{b}+\frac{\Delta x}{2}\right]$ at $\tau=n \Delta t$. The option value is zero in $\left[x_{b}-\frac{\Delta x}{2}, x_{b}\right]$, but assumes positive value in $\left[x_{b}, x_{b}+\frac{\Delta x}{2}\right]$. Suppose $v_{0}^{n}$ is set to be zero as the barrier condition in the numerical algorithm, while in fact $v_{0}^{n}$ is positive because its actual value is $\int_{x_{b}}^{x_{b}+\frac{\Delta x}{2}} v(x, n \Delta t) d x$. Therefore, the setting of $v_{0}^{n}$ to be zero leads to an approximation error of the barrier condition. Indeed, this would lead to under valuation of the barrier option value because a positive quantity has been set artificially to be zero (the same observation is reported in Cheuk \& Vorst, 1996). The simple remedy is to place $x_{b}$ between the nodes $x_{0}$ and $x_{1}$ [see the configuration in Fig. 1(a)]. Now, the interval $\left[x_{0}-\frac{\Delta x}{2}, x_{0}+\frac{\Delta x}{2}\right]$ lies completely inside the knockout region, so the setting of $v_{0}^{n}=0$ in the numerical scheme accurately reflects the discretely monitored barrier condition.

For the continuously monitored case, the best method of placing the boundary node is to set $x_{0}=x_{b}$ [see the configuration in Fig. 1(b)]. By virtue of the odd extension property of the solution, the average value of $v(x, \tau)$ over the interval $\left[x_{0}-\frac{\Delta x}{2}, x_{0}+\frac{\Delta x}{2}\right]$ is close to zero. Hence, with this method of placement of boundary node, $v_{0}^{n}=0$ would be a good approximation of the continuously monitored barrier condition.

\section{Initial Asset Price Close to the Barrier}

It is widely reported in the literature that poor accuracy of barrier-option calculations is observed when the initial asset price is close to the barrier. Rogers and Stapleton (1998) proposed to modify the probability values at nodes next to the barrier. Figlewski and Gao (1999) suggested to construct finer meshes near the barrier to improve the level of accuracy. 


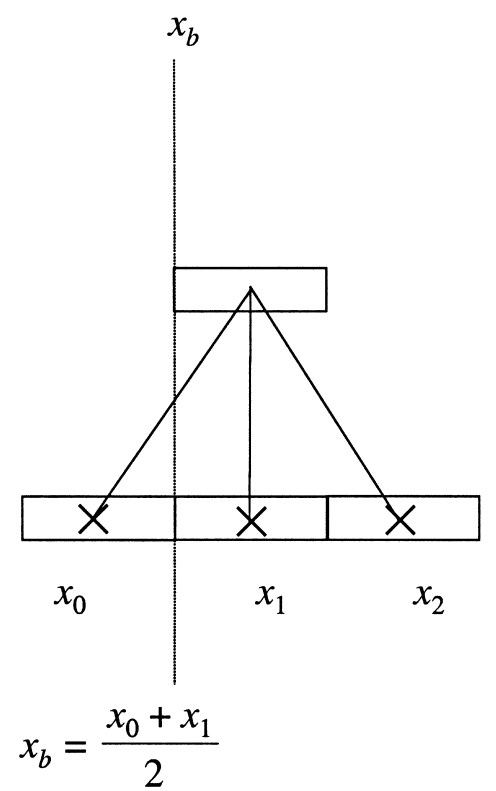

(a) discretely monitored barrier

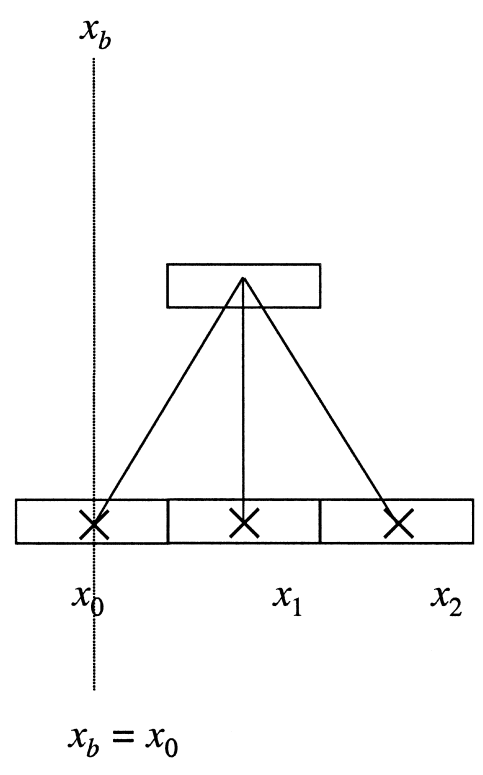

(b) continuously monitored barrier

FIGURE 1

The different methods of placing the barrier node are shown for (a) discretely monitored barrier option, and (b) continuously monitored barrier option. To approximate the discretely monitored barrier condition with better accuracy, it is optimal to place the whole interval $\left[x_{0}-\frac{\Delta x}{2}, x_{0}+\frac{\Delta x}{2}\right]$ to lie completely in the knockout region.

However, Boyle and Tian (1998) observed that the method of spline interpolation of option values at three adjacent nodes is a possible straightforward method to resolve the problem of dealing with initial asset price close to the barrier. Zvan et al. (2000) argued that "initial asset price close to the barrier" is not an issue for implicit schemes because the effects of an instantaneous change to boundary conditions are felt immediately across the entire solution in implicit scheme calculations.

\section{Far-Field Boundary Conditions}

To implement the finite difference calculations of any option-pricing models, it is necessary to prescribe the boundary conditions along all boundaries of the computational domain. This is different from trinomial calculations where only the terminal payoff condition is required. In barrier option calculations, besides the prescription of the numerical boundary condition at the barrier, the far-field numerical boundary condition should also be provided. The far-field boundary condition may be deduced from the nature of the option model. 
Consider a down-and-out barrier call option. As the asset price $S \rightarrow \infty$, the option value $V(S, \tau)$ satisfies either

(i) asymptotic boundary condition: $\lim _{S \rightarrow \infty} V(S, \tau)=S-X e^{-r \tau}$, where $X$ is the strike price, or

(ii) zero second order derivative: $\lim _{S \rightarrow \infty} \frac{\partial^{2} V}{\partial S^{2}}=0$.

Let $V_{M}^{n}$ be the right-most node at the $n$th time level. The first far-field boundary condition leads to the numerical-boundary condition

$$
V_{M}^{n}=e^{x_{M}}-X e^{-r n \Delta t}
$$

In terms of $x=\ln S$, the second far-field boundary condition becomes $\frac{\partial^{2} V}{\partial x^{2}}=\frac{\partial V}{\partial x}$ as $x \rightarrow \infty$. Suppose the following backward difference approximation formulas for the derivatives are used:

$$
\begin{aligned}
& \left.\frac{\partial V}{\partial x}\right|_{(L+M \Delta x, n \Delta t)}=\frac{3 V_{M}^{n}-4 V_{M-1}^{n}+V_{M-2}^{n}}{\Delta x}+O\left(\Delta x^{2}\right) \\
& \left.\frac{\partial^{2} V}{\partial x^{2}}\right|_{(L+M \Delta x, n \Delta t)}=\frac{2 V_{M}^{n}-5 V_{M-1}^{n}+4 V_{M-2}^{n}-V_{M-3}^{n}}{\Delta x^{2}}+O\left(\Delta x^{2}\right),
\end{aligned}
$$

then the far-field numerical boundary condition is given by

$$
2 V_{M}^{n}-5 V_{M-1}^{n}+4 V_{M-2}^{n}-V_{M-3}^{n}=\Delta x\left(3 V_{M}^{n}-4 V_{M-1}^{n}+V_{M-2}^{n}\right) .
$$

The order of accuracy of the approximation is $O\left(\Delta x^{2}\right)$, or equivalently, $O(\Delta t)$.

\section{Sample Calculations}

In the following sample calculations on a continuously monitored downand-out barrier call option, all the "optimal" techniques in the construction of the numerical scheme are adopted. These include:

1. The lattice nodes are placed on the barrier and at the strike.

2. The Black-Scholes analytic formula is used to compute the option value at nodes along the first time level (smoothing the terminal payoff).

3. We use the Lax-Wendroff scheme with the choice of the optimal parameter $\lambda=\sqrt{3}$.

4. We use either scheme (27) or scheme (29) for the far-field numerical boundary condition.

The order of convergence of the numerical option values that can be achieved is examined. The parameter values in the barrier-option 


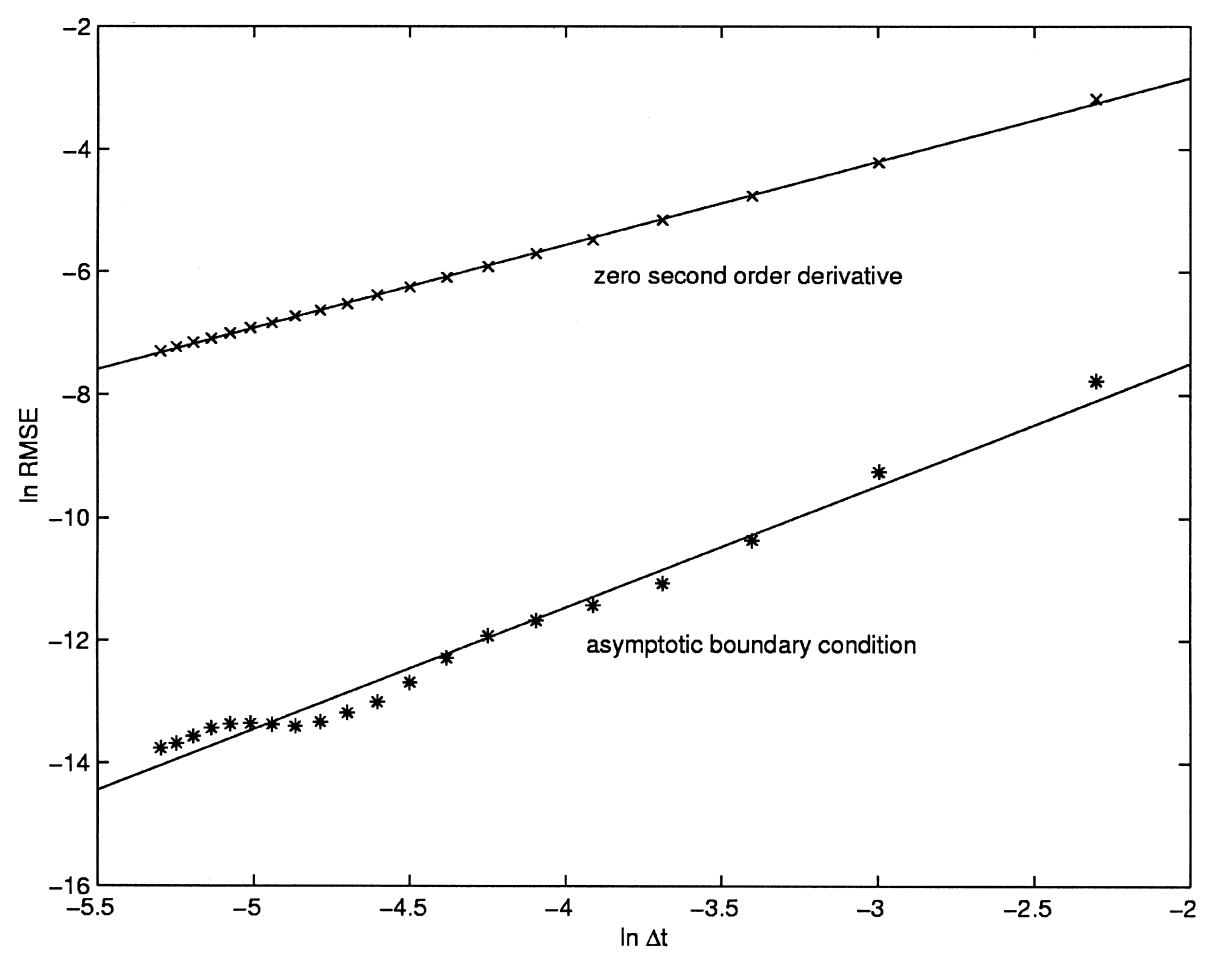

FIGURE 2

The figure plots ln RMSE against ln $\Delta t$, where RMSE denotes the root-meansquared errors in the numerical calculations of the option values of a down-andout barrier call option. The Lax-Wendroff scheme with the choice of the optimal parameter $\lambda=\sqrt{3}$ is used in the calculations, and two different far-field boundary conditions are used. From the regression calculations, the slope of the upper line equals 1.36, while that of the lower line is 1.99. The parameter values used in the barrier option are: $S=100, X=95, r=5 \%, \sigma=30 \%, B=80$, and $\tau=1.0$.

model are: $S=100, X=95, r=5 \%, \sigma=30 \%$, down-and-out barrier $B=80$, and $\tau=1$. In Figure 2, the logarithm of the root mean square errors (ln RMSE) is plotted against the logarithm of the time step $(\ln \Delta t)$ for the numerical barrier-option values obtained using the two far-field numerical boundary conditions. The slope of the linear regression line through the data points corresponding to the use of the asymptotic boundary condition [scheme (27)] is found to be 1.99 , while the slope corresponding to the use of the zero second-order derivative condition [scheme (29)] is found to be 1.36 . With the use of the asymptotic boundary condition and the optimal parameter $\lambda=\sqrt{3}$ in the LaxWendroff scheme, second-order temporal accuracy as predicted by the MEPDE analysis [see eq. (16)] is achieved. The $O(\Delta t)$ approximation of the far-field boundary condition in scheme (29) leads to the degradation of the temporal order of accuracy to a value between one and two. 


\section{Proportional-Step Option Models}

The proportional-step options have the characteristic of proportional amortization of the payoff depending on the time duration of the underlying asset price falling into the knockout region. This class of options retain the advantage of premium savings in barrier-type options while avoiding the discontinuity of the delta at the barrier.

The terminal payoff function of the down-and-out proportional-step call option is defined to be

$$
\exp \left(-\rho \tau_{B}^{-}\right) \max \left(S_{T}-X, 0\right) .
$$

Here, $\rho$ is the killing rate and $\tau_{B}^{-}$is the occupation time in the knockout region defined by

$$
\tau_{B}^{-}=\int_{t_{0}}^{T} H\left(B-S_{t}\right) d t
$$

where $H(x)$ is the Heaviside function, $t_{0}$ is the inception time of the option, and $B$ is the constant down barrier. Linetsky (1999) obtained the following partial differential equation formulation for the value $V(S, \tau)$ of the continuously monitored proportional-step-call option

$$
\begin{gathered}
\frac{\partial V}{\partial \tau}=\frac{\sigma^{2}}{2} S^{2} \frac{\partial^{2} V}{\partial S^{2}}+r S \frac{\partial V}{\partial S}-[r+\rho H(B-S)] V \\
S>0 \text { and } \tau>0 \\
V(S, 0)=\max (S-X, 0)
\end{gathered}
$$

Note that the discount rate equals $r$ when $S>B$ and becomes $r+\rho$ when $S<B$.

The construction of the corresponding Lax-Wendroff scheme for the approximation of eq. (31a) is quite straightforward. The damping factor $d_{j}^{n}$ is introduced, where

$$
d_{j}^{n}=\left\{\begin{array}{ll}
e^{-\rho \Delta t} & \text { if } x_{j}<\ln B \\
1 & \text { otherwise }
\end{array} .\right.
$$

The corresponding Lax-Wendroff scheme then takes the form

$$
\begin{aligned}
V_{j}^{n+1}= & {\left[\frac{c^{2}+\mu+c}{2} d_{j+1}^{n} V_{j+1}^{n}+\left(1-c^{2}-\mu\right) d_{j}^{n} V_{j}^{n}\right.} \\
& \left.+\frac{c^{2}+\mu-c}{2} d_{j-1}^{n} V_{j-1}^{n}\right] e^{-r \Delta t} .
\end{aligned}
$$

The damping factor is applied to $V_{j}^{n}$ when $x_{j}$ lies in the knock-out region. Since $V_{j}^{n}$ represents the average option value over the interval 
$\left[x_{j}-\frac{\Delta x}{2}, x_{j}+\frac{\Delta x}{2}\right]$ at $\tau=n \Delta t$, best accuracy would be resulted if the interval lies wholly in the knockout region. Correspondingly, the barrier level $x_{b}$ is chosen to lie between two layers of nodes [see the configuration in Fig. 1(a)]. Interestingly, this method of placing the nodes for the continuously monitored proportional step option is the same as that for the discretely monitored barrier options.

The comparison of the numerical accuracy of calculating option values of a continuously monitored proportional step call option is performed using the two methods of placing the barrier in the grid lattice, namely, between two layers of nodes and on a layer of nodes. The parameter values used in the calculations are: $S=100, X=95, B=90$, $r=5 \%, \sigma=30 \%, \tau=1.0$, and $\rho=750$. The points labelled with ' $X$ ' in Figure 3 show the option values obtained with the barrier placed between

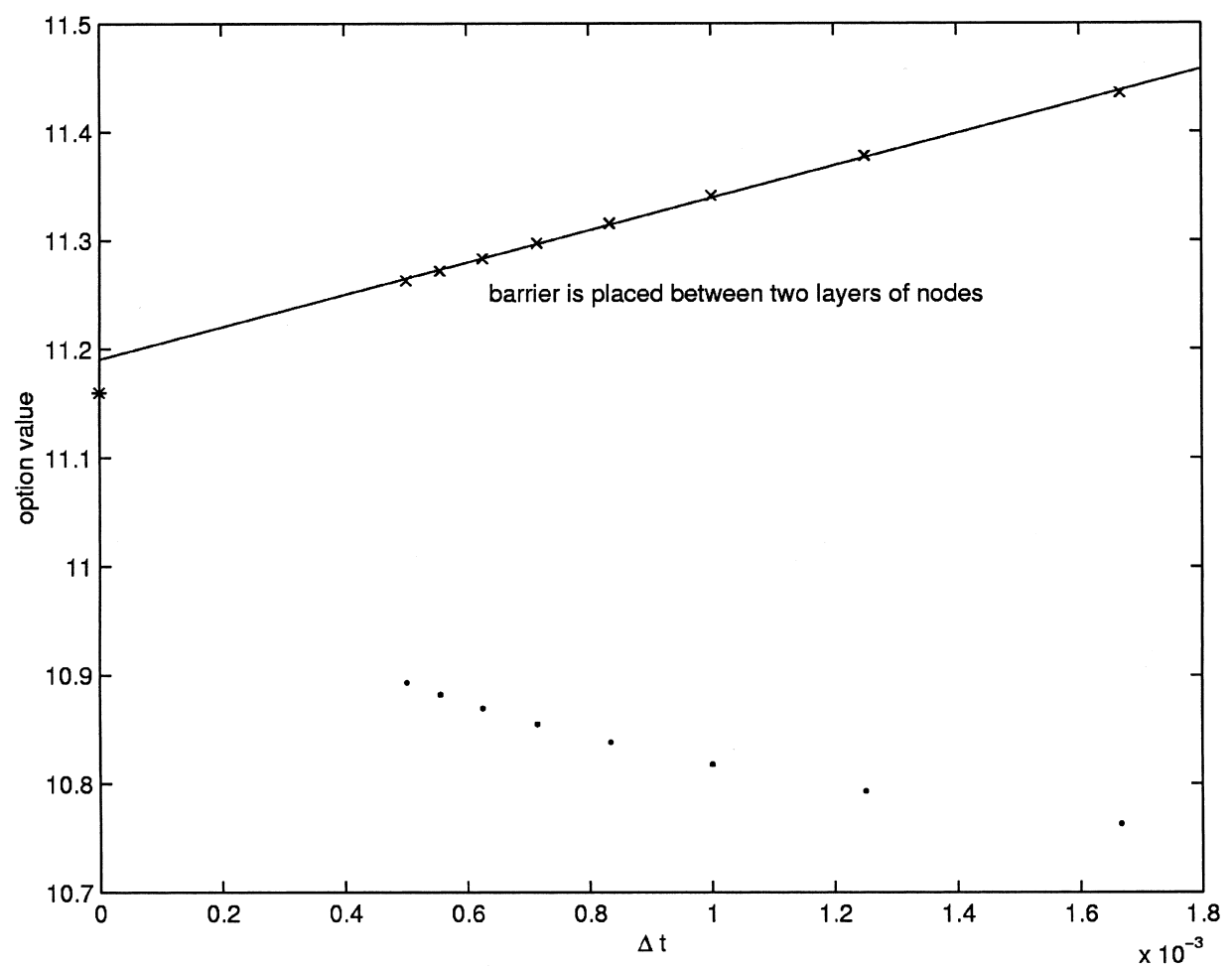

FIGURE 3

The figure reveals the convergence trend of the numerical option values against time-step $\Delta t$ for a continuously monitored proportional-step call option. The crosses $(\times)$ and dots $(\cdot)$ show the results obtained by placing the barrier between two layers of nodes and on a layer of nodes, respectively. The first method apparently gives linear rate of convergence in $\Delta t$, while the second method does not give a clear pattern of convergence. The option value obtained from the analytic formula is 11.16. The parameter values used in the proportional-step call option are: $S=100$, $X=95, r=5 \%, \sigma=30 \%, \rho=750, B=90$, and $\tau=1.0$. 
two layers of nodes. The apparent linear rate of convergence of the numerical option values to the analytic solution as $\Delta t \rightarrow 0$ is revealed.

\section{Lookback Option Models}

Numerical algorithms for pricing lookback options have been proposed by Babbs (2000), Hull and White (1993), Barraquand and Pudet (1999), Cheuk and Vorst (1997). Comparison of these algorithms can be found in the studies by Kat (1995) and Broadie et al. (1999). The Hull-White and Barraquand-Pudet algorithms require an extra dimension to keep track of the current extremum value achieved at a node, so they require higher order of complexity. The Babbs scheme and Cheuk-Vorst scheme avoid the inclusion of an additional statistic that describes the path-dependency feature by constructing a binomial tree for a new state variable, which is defined to be the ratio of the current extremum price to the current asset price. This is equivalent to using the asset price as the numeraire. Broadie et al. (1999) followed the same technique of dimensionality reduction but used the trinomial tree. Barraquand and Pudet (1996) reported square-root rate of convergence of their algorithm, while Babbs and Broadie et al.'s algorithms demonstrate linear rate of convergence in $\Delta t$.

\section{Continuously Monitored Floating Strike Lookback Call Options}

Let $c_{\ell}\left(S, m_{T_{0}}^{t}, t\right)$ denote the price of a continuously monitored European floating strike lookback call option, whose payoff depends on the realized minimum asset price at expiration. Let the realized minimum asset price from $T_{0}$ to $t\left(T_{0} \leq t \leq T\right)$ be denoted by

$$
m_{T_{0}}^{t}=\min _{T_{0} \leq u \leq t} S_{u},
$$

whereby the definition refers to continuous monitoring of the asset price process. The terminal payoff of the floating strike lookback call is defined by

$$
c_{\ell}\left(S_{T}, m_{T_{0}}^{T}, T\right)=S_{T}-m_{T_{0}}^{T} .
$$

The boundary condition at $S=m_{T_{0}}^{t}$ is given by (Goldman, Sosin, \& Gatto, 1979)

$$
\frac{\partial c_{\ell}}{\partial m_{T_{0}}^{t}}=0 \quad \text { at } S=m_{T_{0}}^{t}
$$


With the choice of the following similarity variables:

$$
x=\ln \frac{S}{m_{T_{0}}^{t}} \quad \text { and } \quad v(x, \tau)=\frac{c_{\ell}\left(S, m_{T_{0}}^{t}, t\right) e^{-q \tau}}{S}, \quad \tau=T-t,
$$

the governing equation for $v$ becomes

$$
\frac{\partial v}{\partial \tau}=\frac{\sigma^{2}}{2} \frac{\partial^{2} v}{\partial x^{2}}+\left(r-q+\frac{\sigma^{2}}{2}\right) \frac{\partial v}{\partial x}, \quad x>0, \tau>0 .
$$

Here, $q$ is the dividend yield of the underlying asset. The lookback option model is reduced to a semi-infinite problem with the Neumann boundary condition at $x=0$. Here, $x=0$ commonly is called the reflecting boundary. The auxiliary conditions then become

$$
v(x, 0)=1-e^{-x} \text { and } \quad \frac{\partial v}{\partial x}(0, \tau)=0 .
$$

To approximate the Neumann boundary condition at $x=0$, the following discrete-boundary approximation [known to be $O\left(\Delta x^{2}\right)$ order of accuracy] is adopted:

$$
v_{1}^{n}=v_{-1}^{n},
$$

where $v_{-1}^{n}$ is the option value at a fictitious node one step to the left of the left-end node corresponding to $j=0$. For example, suppose the Lax-Wendroff trinomial scheme is used, the nodal equation at $j=0$ is given by

$$
v_{0}^{n+1}=\left(1-c^{2}-\mu\right) v_{0}^{n}+\left(c^{2}+\mu\right) v_{1}^{n}
$$

The numerical experiments on the European floating strike lookback call option performed by Cheuk and Vorst (1997) are repeated here. The parameter values in the lookback option model are $S=m_{T_{0}}^{t}=100, r=0.04, q=0.07$, and $\tau=0.5$. The comparison of the accuracy of the option calculations using the Cheuk-Vorst scheme, Babbs scheme, and Lax-Wendroff scheme is shown in Table II. The numerical results clearly show the significant improvement on the rate of convergence of numerical option values when the proper discrete boundary approximation is employed. The Lax-Wendroff scheme is shown to have linear rate of convergence in $\Delta t$, and only 50 time steps are required to achieve a percentage error $<0.5 \%$, whereas the Cheuk-Vorst scheme requires more than 10,000 time steps to achieve the same level of accuracy. The Cheuk-Vorst scheme is seen to have only square-root rate of 
TABLE II

Comparison of the Numerical Accuracy of the Lookback Option Values Obtained from the Lax-Wendroff Scheme, Cheuk-Vorst Scheme, and Babbs Scheme

\begin{tabular}{|c|c|c|c|c|c|c|c|}
\hline \multirow[b]{2}{*}{ Volatility } & \multirow{2}{*}{$\begin{array}{c}\text { Numerical } \\
\text { Schemes }\end{array}$} & \multicolumn{5}{|c|}{ Number of Time Steps } & \multirow{2}{*}{$\begin{array}{l}\text { Analytic } \\
\text { Solution }\end{array}$} \\
\hline & & 50 & 100 & 500 & 1000 & 10,000 & \\
\hline \multirow{4}{*}{$\sigma=0.1$} & LW scheme & 4.6691 & 4.6745 & 4.6789 & 4.6794 & 4.6799 & \multirow{3}{*}{4.6799} \\
\hline & CV scheme & 4.24 & 4.37 & 4.54 & 4.58 & 4.65 & \\
\hline & Babbs scheme & 4.6508 & 4.6653 & 4.6770 & 4.6784 & 4.6797 & \\
\hline & LW scheme & 9.7415 & 9.7673 & 9.7870 & 9.7891 & 9.7912 & \multirow{3}{*}{9.7915} \\
\hline \multirow{2}{*}{$\sigma=0.2$} & CV scheme & 8.97 & 9.20 & 9.52 & 9.60 & 9.73 & \\
\hline & Babbs scheme & 9.7362 & 9.7638 & 9.7859 & 9.7887 & 9.7912 & \\
\hline \multirow{3}{*}{$\sigma=0.3$} & LW scheme & 14.5964 & 14.6419 & 14.6785 & 14.6826 & 14.6868 & \multirow{3}{*}{14.6872} \\
\hline & CV scheme & 13.52 & 13.85 & 14.31 & 14.42 & 14.60 & \\
\hline & Babbs scheme & 14.6056 & 14.6464 & 14.6790 & 14.6831 & 14.6868 & \\
\hline
\end{tabular}

The parameter values of the continuously monitored European floating strike lookback call option are: $S=m_{T_{0}}^{t}=100$, $r=0.04, q=0.07$, and $\tau=0.5$.

convergence in $\Delta t$. With this particular set of option parameter values, where $S$ is chosen to be equal to $m_{T_{0}}^{t}$, the reflecting boundary $x=0$ happens to be on a lattice node. Under this scenario, the Babbs scheme also reveals linear rate of convergence in $\Delta t$ and exhibits similar level of accuracy as compared to the Lax-Wendroff scheme.

For the treatment of the Neumann boundary condition, Cheuk and Vorst used the forward difference $\frac{v_{0}^{n}-v_{-1}^{n}}{\Delta x}$ to approximate $\left.\frac{\partial v}{\partial x}\right|_{x=0}$, while here the centered difference $\frac{v_{1}^{n}-v_{-1}^{n}}{2 \Delta x}$ was used as the approximation [see eq. (40)]. The forward difference approximation has only $O(\Delta x)$ accuracy. This explains the apparent $O(\Delta x)=O(\sqrt{\Delta t})$ convergence rate in Cheuk-Vorst's numerical results for the continuously monitored lookback option models.

In general, the reflecting boundary $x=0$ does not fall exactly on a layer of nodes, except that the lattice layout is specifically designed to do so. Babbs proposed to modify the lattice tree along the layer of nodes next to the reflecting boundary. Figure 4 shows the comparison of the numerical option values obtained using the Babbs scheme and the Lax-Wendroff scheme for a continuously monitored floating strikelookback call option. The parameter values used in the lookback option model are: $S=100, m_{T_{0}}^{t}=100 e^{-0.1}, r=4 \%, q=2 \%, \sigma=10 \%, \tau=1.0$. The numerical results demonstrate that the version of the Babbs scheme based on the non-alignment of the nodes on $x=0$ leads to erratic convergence behavior, although the level of accuracy is comparable to that 


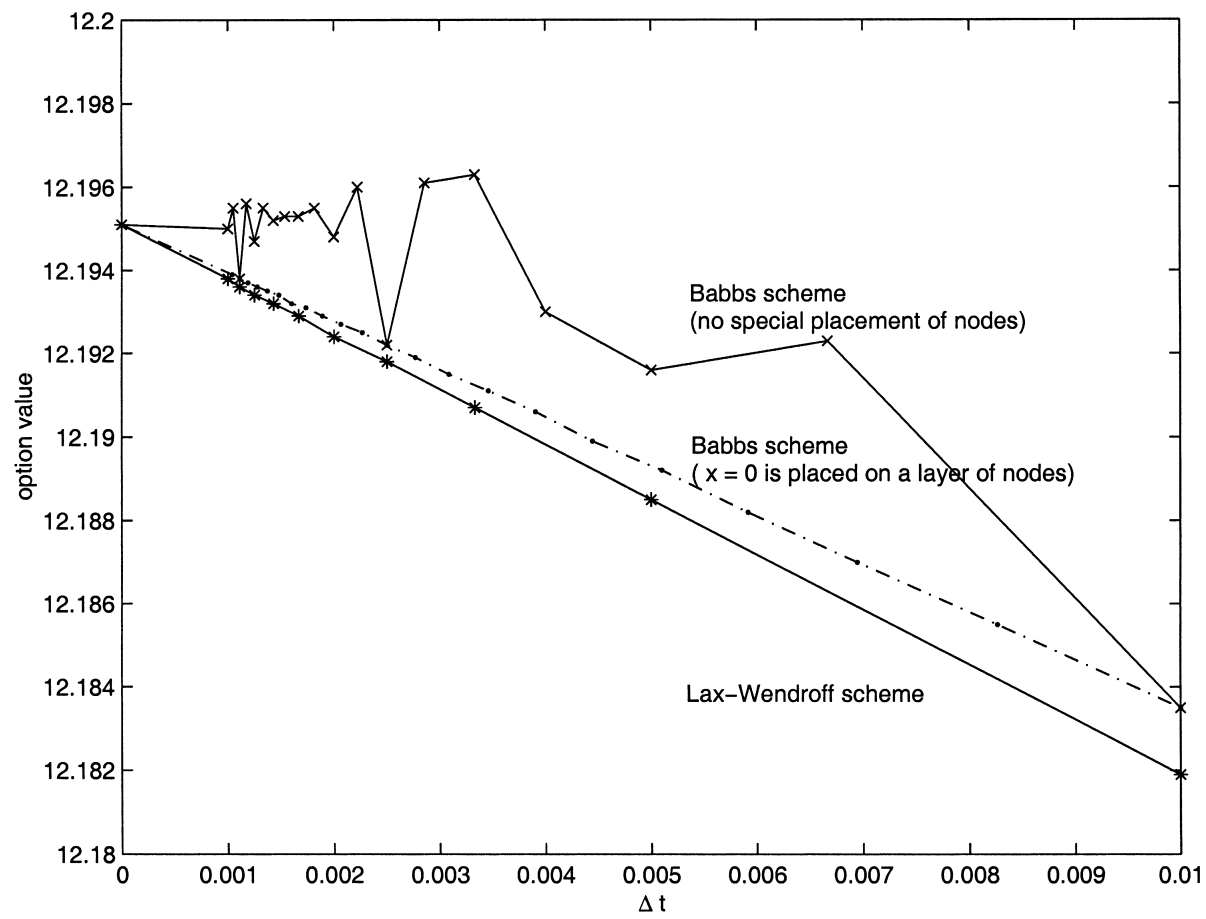

FIGURE 4

The figure compares the accuracy of the numerical option values obtained using the Babbs scheme and the Lax-Wendroff scheme for a continuously monitored floating strike lookback call option. The lowest plot reveals the linear rate of convergence of the Lax-Wendroff scheme. The convergence trend of the numerical option values obtained using the Babbs scheme depends sensibly on the positioning of the reflecting boundary. Linear rate of convergence is exhibited only when the reflecting boundary is placed on a layer of nodes. The parameter values used in the lookback option model are: $S=100, m_{T_{0}}^{t}=100 e^{-0.1}, r=4 \%$,

$$
q=2 \%, \sigma=10 \%, \tau=1.0 \text {. }
$$

of the Lax-Wendroff scheme. If the reflecting boundary $x=0$ is placed exactly on a layer of nodes, then the numerical option values from the Babbs scheme exhibit linear rate of convergence in $\Delta t$. In the present Lax-Wendroff scheme calculations, spline interpolation is used to obtain the option value at a given value of $x$ if that $x$ does not fall on a node. The numerical option values from the Lax-Wendroff scheme clearly reveal the linear rate of the convergence, although the chosen value of $x$ in the numerical calculations is quite close to the reflecting boundary.

\section{Discretely Monitored Floating Strike Lookback Call Options}

For the discretely monitored floating strike lookback call options, the realized minimum is updated only on a monitoring instant. Let $m_{\ell}$ 
denote the realized minimum at the $\ell$ th monitoring instant. Similarly, let $x=\ln \frac{S}{m_{\ell}}$ and $v(x, \tau)=\frac{c_{\ell}\left(S, m_{\ell}, t\right) e^{-q \tau}}{S}$, which are applicable within the $\ell$ th monitoring instants [including the $\ell$ th instant but excluding the $(\ell+1)$ th instant]. The governing equation for $v$ remains the same as in eq. (38), except that the domain of $x$ is now $(-\infty, \infty)$ instead of $(0, \infty)$. The initial condition remains to be $v(x, 0)=1-e^{-x}$, but the Neumann boundary condition $\frac{\partial v}{\partial x}(0, \tau)=0$ is applied only at those time steps corresponding to monitoring instants.

For numerical calculations, the usual trinomial calculations are performed as that of a vanilla option at time levels not corresponding to a monitoring instant. However, when the $n$th time level happens to be on a monitoring instant, the boundary condition $\frac{\partial v}{\partial x}(0, \tau)=0, x \leq 0$ is implemented by setting the numerical option values to the left of $x=0$ to be

$$
v_{j}^{n}=v_{0}^{n}, \quad j=-1,-2, \ldots
$$

Recall that there are two choices of positioning the nodes relative to $x=0$, either placing $x=0$ at $x_{0}$ or between $x_{0}$ and $x_{1}$. In either case, $v_{0}^{n}=v_{-1}^{n}=v_{-2}^{n}=\ldots$. The Lax-Wendroff scheme at $j=0$ on a monitoring instant is given by

$$
v_{0}^{n+1}=\frac{c^{2}+\mu+c}{2} v_{1}^{n}+\left(1-\frac{c^{2}+\mu+c}{2}\right) v_{0}^{n}
$$

for both positioning methods. Due to the zero-derivative condition applied at $x=0$, the lookback-option value is expected to level off when $x$ is close to $x_{0}$. Therefore, the numerical accuracy of the approximation of the auxiliary condition becomes almost insensitive to the positioning of the nodes relative to $x=0$. Both positioning methods are expected to give the same rate of convergence of the numerical option values for the discretely monitored lookback options.

Figure 5 shows the plots of numerical option value against timestep $\Delta t$ obtained from the Lax-Wendroff scheme. The parameter values in the discretely monitored floating strike lookback call option are: $S=100, m_{T_{0}}^{t}=95, r=5 \%, q=0, \sigma=25 \%, \tau=0.5$, and the number of monitoring instants is 20 . For both positioning methods, the numerical option values exhibit linear rate of convergence in $\Delta t$. Among barrier-type options and lookback options, the discretely monitored lookback options are the only class where the rate of convergence of numerical option values is insensitive to the method of positioning the nodes. 


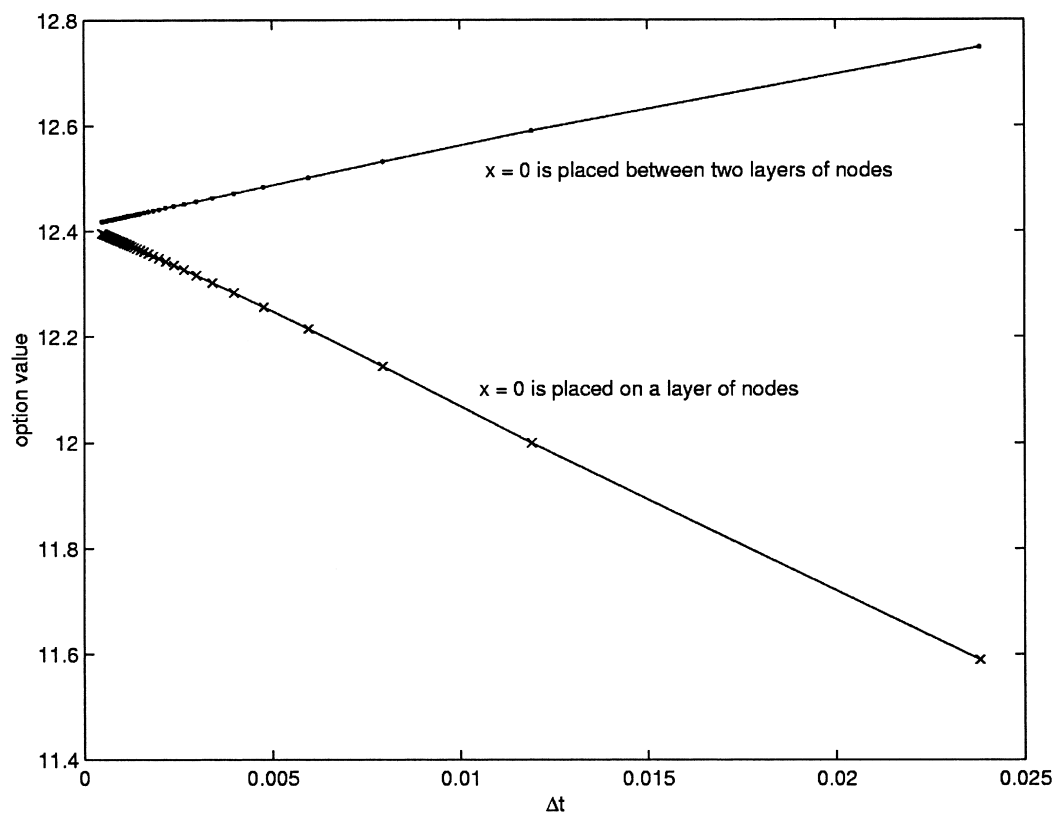

FIGURE 5

The figure shows the plots of numerical option value against time step

$\Delta t$ for a discretely monitored floating strike lookback call option.

The numerical scheme used is the Lax-Wendroff scheme. The upper (lower) plot shows the numerical option values obtained by positioning $x=0$ between two layers (on a layer) of nodes. Both plots reveal the linear rate of convergence in $\Delta t$. The parameter values used in the lookback option model are: $S=100, m_{T_{0}}^{t}=95, r=5 \%$, $\sigma=25 \%, \tau=0.5$, and the number of monitoring instants is 20 . The extrapolated option value at $\Delta t=0$ is 12.41 for both methods of positioning.

\section{SUMMARY AND CONCLUSION}

The trinomial schemes and the explicit finite difference schemes are seen to belong to the same class of numerical schemes, although they are derived by quite different approaches. For example, the KamradRitchken trinomial scheme and the Forward-Time-Centered-Space difference scheme are essentially equivalent. All numerical schemes are shown to depend on the diffusion parameter $\mu$ and the drift parameter $c$. The class of two-level explicit and implicit schemes can be analyzed within a unified framework. The order of truncation error of a numerical scheme can be revealed explicitly by analyzing its modified equivalent partial differential equation. Most common numerical schemes are shown to be first-order time accurate, except that the Lax-Wendroff scheme becomes second-order time accurate with the choice of the optimal parameter $\lambda=\sqrt{3}$. 
The order of convergence of numerical option values may be degraded due to the non-differentiability of the terminal payoff function and inaccurate prescription of numerical boundary conditions. By carefully choosing the near-field and far-field numerical boundary conditions and positioning the strike price at a node, it is demonstrated that the optimal Lax-Wendroff scheme does exhibit second-order temporal accuracy.

The linkage is established between the non-negativity of the coefficients in the numerical scheme and the oscillation phenomena of the numerical option values. In the CIR interest-rate process, the volatility of interest rate becomes vanishingly small when the interest rate tends to zero. This may cause the diffusion parameter to be smaller than the drift parameter. To avoid the occurrence of oscillations, the lower bound on the interest rate value in the bond price calculations have to be restricted such that no negative coefficients in the numerical scheme appear.

The positioning of the nodes relative to the barrier (absorbing or reflecting) has profound influence on the order of convergence of numerical option values (see Table III for a summary). The reason why one should place the barrier between two layers of nodes for the discretely monitored barrier options but on a layer of nodes for the continuously monitored counterparts is explained. However, it is demonstrated that the barrier should be positioned between two layers of nodes for the continuously monitored proportional-step options in order to achieve better rate of convergence.

For continuously monitored lookback options, the reflecting boundary should be positioned on a layer of nodes in order to achieve $O(\Delta t)$ accuracy. If the Neumann boundary condition is approximated only to $O(\Delta x)$ accuracy, the order of accuracy of the numerical option values

TABLE III

A Summary of the Optimal Choices of the Positioning of the Nodes Relative to the

Barrier (Absorbing or Reflecting) to Achieve the Linear Rate of Convergence for

Different Types of the Path-Dependent Options and Monitoring Frequencies

\begin{tabular}{|c|c|c|c|}
\hline & & $\begin{array}{c}\text { Barrier Between Two } \\
\text { Layers of Nodes }\end{array}$ & $\begin{array}{c}\text { Barrier on a Layer } \\
\text { of Nodes }\end{array}$ \\
\hline Barrier options & $\begin{array}{l}\text { Continuously monitored } \\
\text { Discretely monitored }\end{array}$ & $\boldsymbol{V}$ & $\checkmark$ \\
\hline Lookback options & $\begin{array}{l}\text { Continuously monitored } \\
\text { Discretely monitored }\end{array}$ & $\checkmark$ & $\checkmark$ \\
\hline Proportional-step options & Continuously monitored & $\checkmark$ & \\
\hline
\end{tabular}


reduces to $O(\sqrt{\Delta t})$. This is because the relative order of magnitude $O(\Delta x)=O(\sqrt{\Delta t})$ is observed in the option pricing calculations. Interestingly, for the discretely monitored lookback options, the order of accuracy of numerical option values is insensitive to the method of positioning the reflecting boundary relative to the layers of nodes.

\section{BIBLIOGRAPHY}

AitSahlia, F., \& Carr, P. (1997). American options: A comparison of numerical methods. In L. C. G. Rogers \& D. Talay (Eds.), Numerical methods in finance (pp. 67-87). Cambridge, UK: Cambridge University Press.

Babbs, S. (2000). Binomial valuation of lookback options. Journal of Economic Dynamics \& Control, 24, 1499-1525.

Barraquand, J., \& Pudet, T. (1996). Pricing of American path-dependent contingent claims. Mathematical Finance, 6, 17-51.

Black, F., \& Scholes, M. (1973). The pricing of options and corporate liabilities. Journal of Political Economy, 81, 637-659.

Broadie, M., \& Detemple, J. (1997). Recent advances in numerical methods for pricing derivative securities. In L. C. G. Rogers \& D. Talay (Eds.), Numerical methods in finance (pp. 43-66). Cambridge, UK: Cambridge University Press.

Broadie, M., Glasserman, P., \& Kou, S. (1999). Connecting discrete and continuous path-dependent options. Finance and Stochastics, 3, 55-82.

Boyle, P. P. (1988). A lattice framework for option pricing with two state variables. Journal of Financial and Quantitative Analysis, 23, 1-12.

Boyle, P. P., Broadie, M., \& Glasserman, P. (1997). Monte Carlo methods for security pricing. Journal of Economic Dynamics and Control, 21, 1267-1321.

Boyle, P. P., \& Tian, Y. (1998). An explicit finite difference approach to the pricing of barrier options. Applied Mathematical Finance, 5, 17-43.

Büttler, H. J. (1995). Evaluation of callable bonds: Finite difference methods, stability and accuracy. Economic Journal, 105, 374-384.

Cheuk, T. H. F., \& Vorst, T. C. F. (1996). Complex barrier options. Journal of Derivatives, 8-22.

Cheuk, T. H. F., \& Vorst, T. C. F. (1997). Currency lookback options and observation frequency: A binomial approach. Journal of International Money and Finance, 16, 173-187.

Cox, J. C., Ross, S., \& Rubinstein, M. (1979). Option pricing: A simplified approach. Journal of Financial Economics, 7, 229-264.

Crank, J., \& Nicolson, P. (1947). A practical method for numerical evaluation of solutions of partial differential equations of the heat conduction type. Proceedings of the Cambridge Philosophical Society, 43, 50-67.

Figlewski, S., \& Gao, B. (1999). The adaptive mesh model: A new approach to efficient option pricing. Journal of Financial Economics, 53, 313-351.

Geske, R., \& Johnson, H. E. (1984). The American put option valued analytically. Journal of Finance, 39, 1511-1524. 
Goldman, M. B., Sosin, H. B., \& Gatto, M. A. (1979). Path dependent options: Buy at the low, sell at the high. Journal of Finance, 34, 1111-1127.

Heston, S., \& Zhou, G. (2000). On the rate of convergence of discrete-time contingent claims. Mathematical Finance, 10, 53-75.

Huang, J. A., Subrahmanyam, M. G., \& Yu, G. G. (1996). Pricing and hedging American options: A recursive integration method. Review of Financial Studies, 9, 277-300.

Hull, J., \& White, A. (1993). Efficient procedures for valuing European and American path-dependent options. Journal of Derivatives, 21-31.

Kamrad, B., \& Ritchken, P. (1991). Multinomial approximating models for options with $k$ state variables. Management Science, 37, 1640-1652.

Kat, H. (1995). Pricing lookback options using binomial trees: An evaluation. Journal of Financial Engineering, 4, 375-397.

Kwok, Y. K. (1992). Stability analysis of six-point finite difference schemes for the constant coefficient convective-diffusion equation. Computers and Mathematics with Applications, 23, 3-11.

Lax, P. D., \& Wendroff, B. (1960). Systems of conservation laws. Communications in Pure and Applied Mathematics, 13, 217-237.

Linetsky, V. (1999). Step options. Mathematical Finance, 9, 55-96.

MacMillan, L. W. (1986). Analytic approximation for the American put option. Advances in Futures and Options Research, 1, 119-139.

Rogers, L. C. G., \& Stapleton, E. J. (1998). Fast accurate binomial pricing. Finance and Stochastics, 2, 3-17.

Steiner, M., \& Wallmeier, M. (1999). Pricing near the barrier: The case of discrete knock-out options. Journal of Computational Finance, 3, 69-90.

Tavella, D. (2000, August). Stretching for accuracy. Risk, 83.

Thomas, J. W. (1995). Numerical partial differential equations. New York: Springer-Verlag.

Tian, Y. (1993). A modified lattice approach to option pricing. Journal of Futures Markets, 13, 563-577.

Tian, Y. (1997). Pricing options with discontinuous barriers. Journal of Financial Engineering, 6, 193-216.

Tian, Y. (1999). A flexible binomial option pricing model. Journal of Futures Markets, 19, 817-843.

Vetzal, K. R. (1998). An improved finite difference approach to fitting the initial term structure. Journal of Fixed Income, 62-81.

Zvan, R., Vetzal, K. R., \& Forsyth, P. A. (1998a, March) Swing low, swing high. Risk, $71-75$.

Zvan, R., Forsyth, P. A., \& Vetzal, K. R. (1998b). Robust numerical methods for PDE models of Asian options. Journal of Computational Finance, 1, $39-78$.

Zvan, R., Vetzal, K. R., \& Forsyth, P. A. (2000). PDE methods for pricing barrier options. Journal of Economic Dynamics and Control, 24, 1563-1590. 
